\title{
DROHNEN-ANSAMMLUNGEN BEI NESTERN DER STACHELLOSEN BIENE SCAPTOTRIGONA POSTICA*
}

\author{
Elisabeth ENGELS und Wolf ENGELS \\ Lehrstuhl Entwicklungsphysiologie, Institut für Biologie III (Zoologie), \\ Universität Tübingen, Auf der Morgenstelle 28, D - 7400 Tübingen, Germany, \\ und Departamento de Genética, Universidade de Sāo Paulo, Campus de Ribeirāo Preto, \\ BR - 14100 Ribeirāo Preto, S.P., Brasil
}

\section{ZUSAMMENFASSUNG}

Es wird eine kurze Einführung in die Fortplanzungsbiologie stachelloser Bienen gegeben, über die erst relativ wenig Untersuchungen vorliegen. Hier wird über Drohnen-Ansammlungen bei der brasilianischen Art Scaptotrigona postica berichtet. Die Aggregationen bestehen aus einigen wenigen Männchen bis zu vielen Tausend. Bei günstigem Wetter sind sie tagsüber in Nestnähe auf Sitzplätzen anzutreffen die wahrscheinlich mit einem Aggregations-Pheromon markiert werden. Die Männchen-Ansammlung vor einem Nest besteht aus Drohnen von vielen Kolonien eines Areals. Gezeichnete Drohnen können über längere Zeit in derselben Aggregation beobachtet werden. Benachbarte Männchen-Gruppen durchmischen sich kaum. Drohnen reagieren auf von Arbeiterinnen abgegebenes Alarm-Pheromon, daneben verfïgen sie wahrscheinlich noch über einen eigenen Alarmduft.

Die Drohnen werden offensichtlich durch ein weibliches Sexual-Pheromon angelockt, das von im Volk vorhandenen unbegatteten Jungköniginnen abgegeben wird. Eine solche PaarungsStrategie ähnelt dem Verhalten der Männchen bei vielen Arten solitärer Bienen und Hummeln, bei denen junge Weibchen am Nest erwartet werden.

Die Paarungsbiologie der stachellosen Bienen erscheint unter Evolutions-Gesichtspunkten daher weniger stark abgeleitet als die der Honigbienen. Bei beiden hoch-eusozial organisierten Apiden sind Männchen-Gruppen heterogener Herkunft Teil eines Fremdpaarung sichernden Fortpflanzungs-Systems. Obwohl sie auf völlig unterschiedliche Weise zustande kommen, haben die nestnahen Drohnen-Ansamminngen der stachellosen Bienen und die nestfernen DrohnenSammelplätze der Honigbienen somit reproduktionsbiologisch analoge Funktionen.

\section{EINLEITUNG}

Bei Honigbienen (Apinae) wurden zahlreiche fortpflanzungsphysiologische Strategien beschrieben, die mit hoher Wahrscheinlichkeit dazu führen, dass eine

* Friedrich Ruttner, dem Pionier auf dem Gebiet der Paarungsbiologie der Bienendrohnen, zum 70. Geburtstag am 15.5.1984 gewidmet. 
Geschwister-Paarung verhindert wird und dennoch entstehende, minderfertile Homozygote ausgeschaltet werden. Kopulationen finden niemals im Nest, sondern nur im freien Fluge statt, vorzugsweise auf entfernten Drohnensammelplätzen (RutTNER and RutTner, 1972). Da eine Königin von mehreren Drohnen begattet wird (RuTrner, 1956), enthält die Spermatheca einen Samenvorrat, in dem viele der in der jeweiligen Population vorhandenen Sexallele vertreten sind (ADAMS et al., 1977). Unter natürlichen Bedingungen sind dip.oide Drohnen daher selten, sic werden zudem bereits als Junglarven von den Ammenbienen eliminiert (WOYKE, 1963).

$\mathrm{Ob}$ bei anderen hoch-eusozialen Hymenopteren ähnliche Fortpflanzungssysteme existieren, ist noch weitgehend unbekannt (EICKworT und GinsberG, 1980). Auch über die Paarungsbio'ogie stachelloser Bienen (Meliponinae) gibt es erst relativ wenige Untersuchungen (vgl. NoGUEIRA-Neto, 1970 ; Wilson, 1971 ; Michener, 1974 ; Wille, 1983). Bei einigen südamerikanischen Arten wurden das Verhalten der Jungkönigin (KERR und Krause, 1950 ; IMPERATriz-FonsECA et al., 1975 ; IMPERATRIZ-FonSECA, 1977), ihr Versteck-Territorium (NociUEIRANeto, 1958 ; Juliani, 1962 ; Terada, 1974), Königinnen-Ersatz (Silva et al., 1972 ; Imperatriz-Fonseca, 1978), Hochzeitsflug (Kerr und KraUse, 1950 ; Kerr et al., 1962 ; Imperatriz-Fonseca, 1977), Schwärmen (Schwarz, 1948 ; Nogueira-Neto, 1954 ; Kerr et al., 1962 ; Terada, 1972 ; ImperatrizFonseCA, 1977) und die Erzeugung von Drohnen (BEIG, 1972 ; BEgo, 1982) beschrieben. Das Verhaltens-Inventar der Drohnen ist bei stachellosen Bienen vielseitiger als bei Honigbienen. Junge Männchen beteiligen sich an Arbeiten im Nest ; später verlassen die Drohnen die Mutterkolonie und streifen umher, sie besuchen Blüten und nächtigen im Freien (KerR et al., 1962 ; SaKaGami, 1982). Zeitweise bilden die Drohnen in der Nähe von Nestern Aggregationen. Ihre Form und Dauer sind artspezifisch, sie werden als Paarungs-Schwarm oder DrohnenAnsammlung beze chnet (vgl. Michner, 1974; SAKaGAmi, 1982). Nur Kerr et al. (1962) stellten einige Beobachtungen und Untersuchungen zu diesem interessanten Phänomen an, Abbildungen wurden noch nie veröffentlicht. Wir berichten hier über Männchen-Ansammlungen bei Scaptotrigona postica (LATrEILLE, 1807), einer brasilianischen Art des Tribus Trigonini.

\section{MATERIAL UND METHODEN}

Scaptotrigona postica (syn. : Trigona, Nannotrigona, vgl. SCHWARZ, 1948 ; Moure, 1961 ; Wille, 1983) nistet in Baumhöhlen. Unsere Beobachtungen wurden an wilden Kolonien im Staat São Paulo und an Laborvölkern durchgeführt, die wir seit 1973 in Brasilien und in Deutschland halten. Sie sind in Beuten nach Nogueira-Neto (1970) oder in beheizten Kästen (modifiziert nach SaKagami, 
1966) untergebracht und werden mit Zuckerwasser und Pollen gefüttert. Farbmarkierungen wurden nach kurzer Kältenarkose durchgeführt.

\section{ERGEBNISSE}

Drohnen-Ansammlungen bilden sich bei Scaptotrigona postica stets in Nas.nähe und als temporäre Erscheinung. Sie können aus wenigen, aber auch aus einigen Tausend Männchen bestehen, die dann dicht gedrängt und übereinander sitzen (Abb. 1).

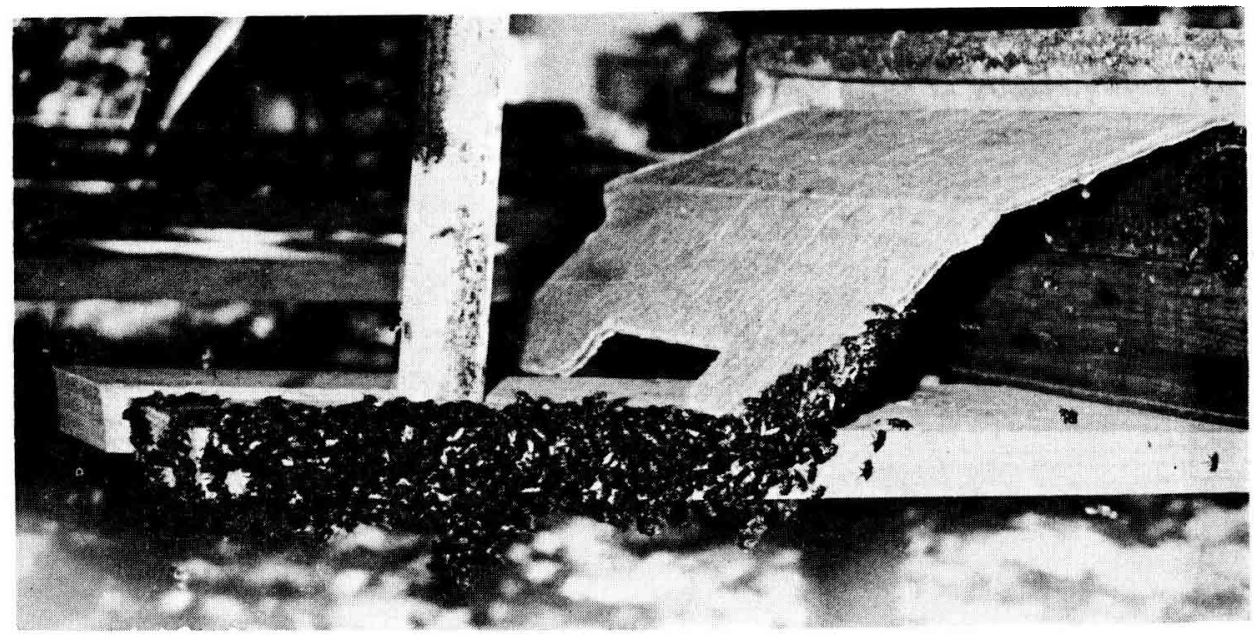

Авв. 1. - Ansammlang von ca. 2000 Drohnen neben einem Versuchsvolk von Scaptotrigona postica

Die Männchen sitzen auf dam Stellbrett eines überdachten Bienenstandes und einem Karton, mit dem der aus 2 flachen Zargen bestehende Bienenkasten abgedeckt ist. Abstand bis zam Nesteingang (rechts ausserhalb des Bildes) ca. $75 \mathrm{~cm}$.

FIG. 1. - Aggregation of ca. 2000 drones aside an experimental colony of Scaptotrigona postica

The males are sitting on the board of a roofed apiary and on a cartboard used to cover the hive which consists of two flat frames. The distance to the nest entrance (right hand outside the picture) is ca. $75 \mathrm{~cm}$.

Drohnen werden bei dieser Spezies das ganze Jahr ïber erzeugt (vgl. Bego, 1982). In unseren Laborvölkern in Europa schlüpften auch während des Winters, wenn ein Freiflug nicht möglich ist, fortlaufend Männchen. In Brasilien verlassen die Drohnen ihre Mutterkolonie im Alter von etwa 2 Wochen. Markierte Männchen wurden später nicht in Drohnen-Ansammlungen vor dem Volk, in dem sie auf- 


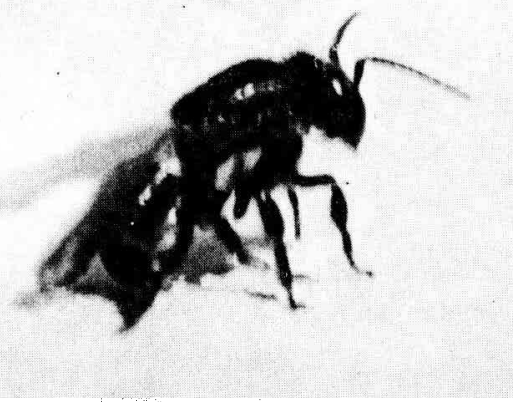

Авв. 2. - Einzelnes Männchen in Nestnähe sitzend, in der für Drohnen typischen Haltung mit aufgerichtetem Vorderkörper und vorgestreckten Antennen

Männchen sind ca. $6 \mathrm{~mm}$ lang und wiegen etwa $15 \mathrm{mg}$.

FIG. 2. - A single male sitting close to the nest in the position typical for clrones, upright and antennae stretched forward

The size of males is ca. $6 \mathrm{~mm}$ the weight approximately $15 \mathrm{mg}$.

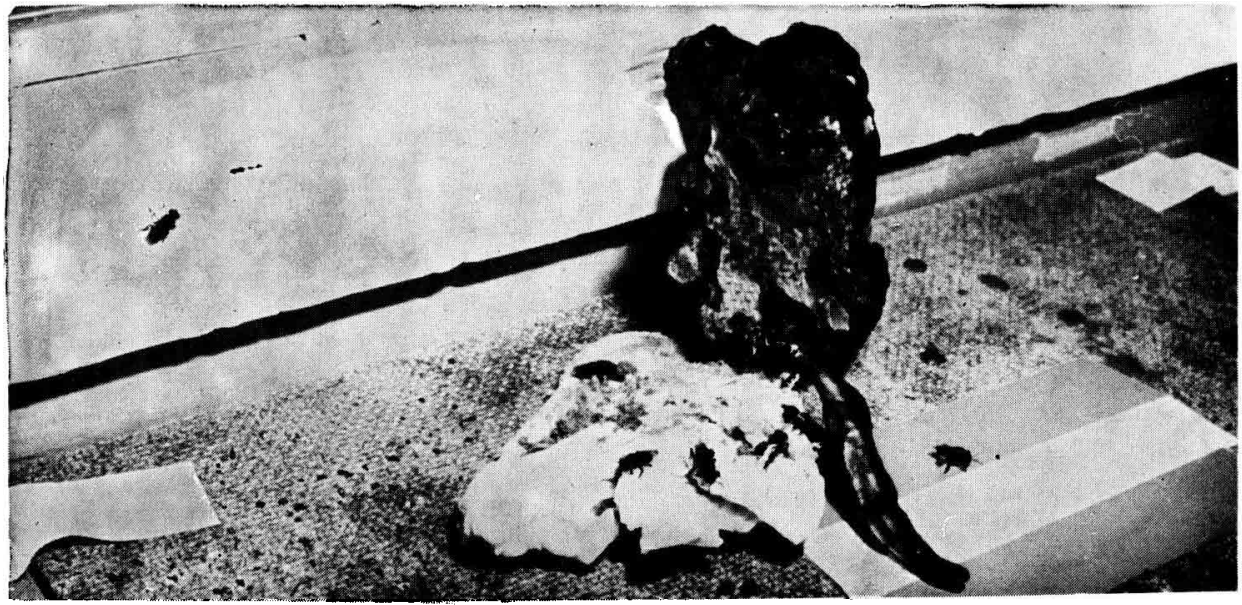

Авв. 3. - Drohnen-Ansammlung in Deutschland

Nur wenige Männchen haben sich in der Nähe des trichterförmigen Flugloches eingefunden.

FIG. 3. - Drone aggregation in Germany

Only a few males appeared around the funnel-like nest entrance. 
gewachsen sind, beobachtet. In Deutschland entstanden vor unseren VersuchsVölkern, auch wenn diese recht stark waren, niemals grössere MännchenAnsammlungen (Abb. 3).

Einzelne Männchen in typischer Haltung mit aufgerichtetem Vorderkörper und vorgestreckten Antennen (Abb. 2) sitzen oft in der Umgebung cines Nesteingangs (Abb. 3). Daraus können im Laufe einiger Tage lockere MännchenGruppen (Abb. 4) und dann individuenreiche Drohnen-Ansammlungen entstehen (Abb. 1).

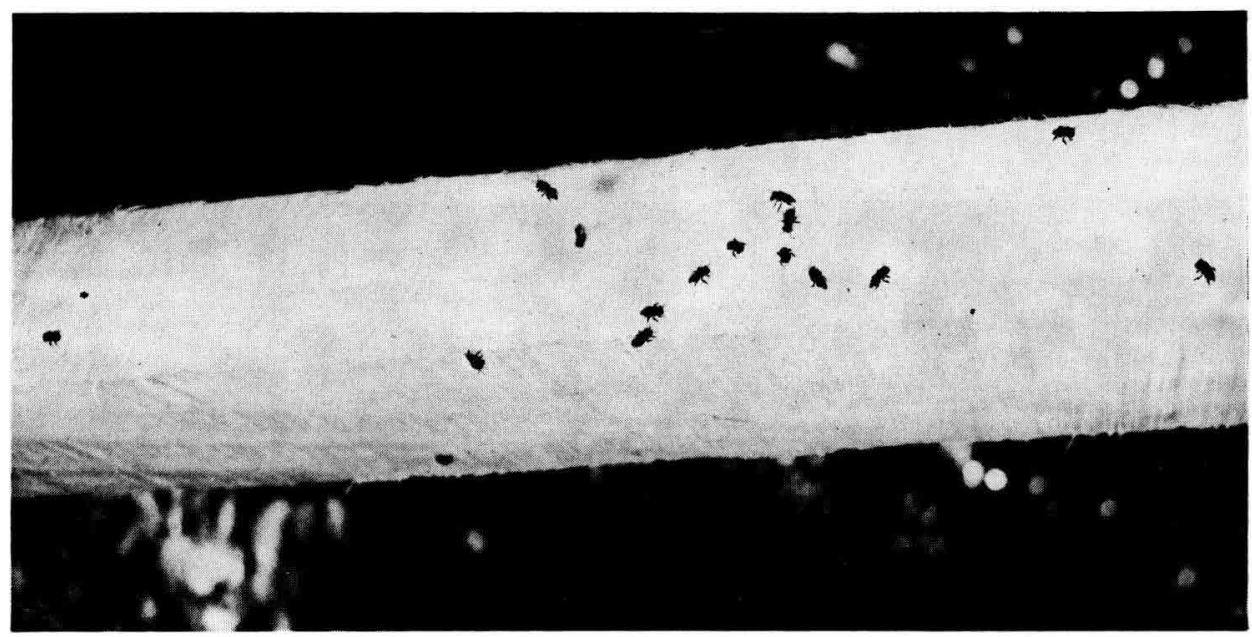

Aвв. 4. - Aggregation einiger Drohnen vor einem Bienenstand

FiG. 4. - Several drones aggregating in front of an apiary

Bei wilden Kolonien sahen wir Männchen-Gruppen auf belaubten Ästen in 20-80 cm vom Flugloch sitzen. Bei Versuchs-Völkern in freistehenden Bienenkästen enstanden die Drohnen-Ansammlungen entweder auf dem Kasten selbst oder seiner Unterlage bzw. Abdeckung (Abb. 1). An einem Volk sind gelegentlich 2 oder 3 Männchen-Gruppen zu sehen, etwa beiderseits des Flugloches, jedoch nie unmittelbar davor. Sind in der Nähe des Nesteingangs keine geeigneten Sitzplätze vorhanden oder stehen mehrere Versuchsvölker dicht nebeneinander, so können sich die Drohnen-Ansammlungen auch in einigen Metern Entfernung, z.B. auf einem Busch, bilden. Vor benachbarten Nestern bildet sich oft nur eine Ansammlung.

Vor starken Kolonien sind Drohnen-Ansammlungen oft über Wochen hinweg zu beobachten. Dabei werden die Sitzplätze recht konstant beibehalten. Auf gross- 
flächigen Unterlagen wie den Stellbrettern eines Bienenstandes (Abb. 1) kann es allerdings von Tag zu Tag zu kleinen Verschiebungen kommen. Entfernt man den Sitzplatz, indem man etwa die entsprechenden Blätter eines Strauches abschneidet, so bildet sich erst im Verlauf einiger Stunden in der Nähe eine neue Aggregation. Verlagert man den Sitzplatz oder Teile desselben, so fliegen die Männchen zum Teil die neue Position an, jedoch nur bei seitlichen Verschiebungen bis ca. $1 \mathrm{~m}$. Deckt man den Sitzplatz mit einem Stück Papier oder einem grossen Blatt ab, so sind die Drohnen desorientiert und lassen sich erst nach einer Weile in der Nähe nieder.

Bei kleinen Völkern kommt es manchmal nur ein paar Tage lang zu einer Männchen-Ansammlung. An Ablegern mit Jungköniginnen stellten wir fest, dass die Drohnen-Aggregationen (Abb. 5) etwa eine Woche lang bestanden und sich einige Tage, bevor die Jungkönigin mit der Eiablage begann, aufï̈sten.

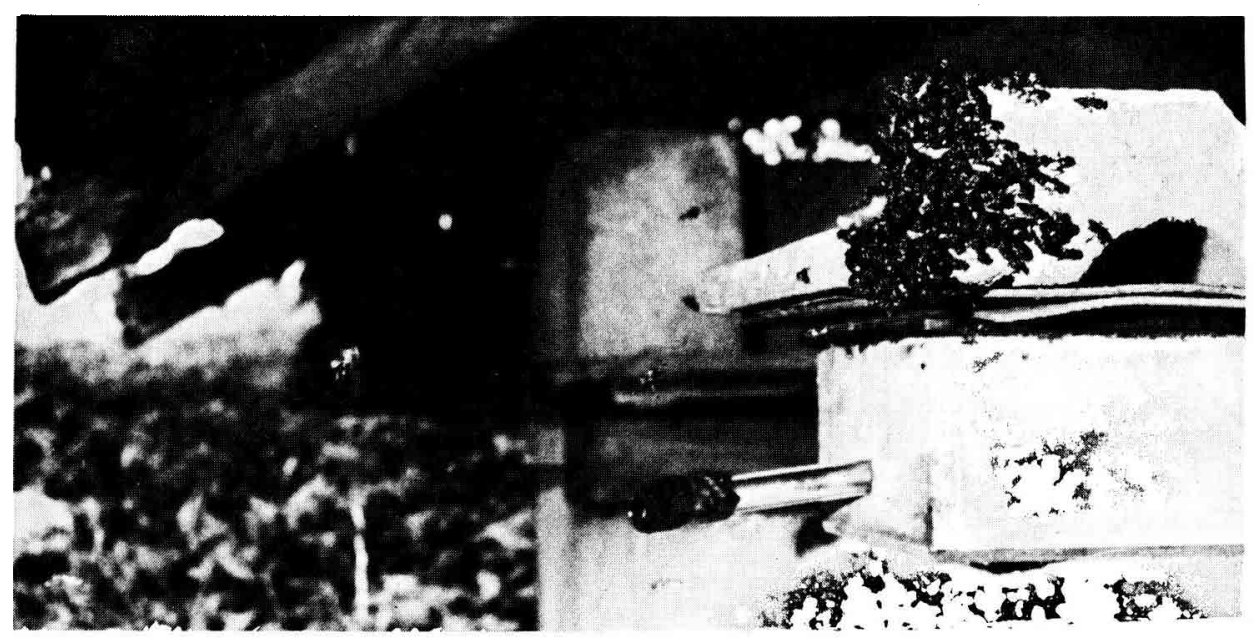

Aвв. 5. - Drohnen-Ansammlang an einem Ableger-Völkchen mit einer Jungkönigin

Fici. 5. - Drone aggregation at a nucleas with a birgin queen

Die Drohnen-Ansammlungen bestehen nur tagsüber. Bei sonnigem und warmen Wetter erfolgt der Anfiug frühmorgens, an regnerischen und kühlen Tagen erst während der Vormittagsstunden. An kalten Tagen bleiben die Männchen ganz oder teilweise aus. Oft sind am Nachmittag die Aggregationen deutlich stärker als am Vormittag. 1-2 Std. vor Sonnenuntergang fliegen die Männchen ab, und zwar einzeln.

Bei Scaptotrigona postica ist die Flugaktivität einer Drohnen-Ansammlung in der Regel gering. Die anfliegenden Männchen setzen sich meistens für längere 
Zeit. Der Flugbetrieb ist in den frühen Nachmittagsstunden am stärksten. Wenn an heissen Tagen reger Zuflug herrscht, bilden sich zuweilen $5-10 \mathrm{~cm}$ lange Trauben von Männchen. Sie reissen in kurzem Abstand ab, fallen zu Boden und bilden sich aus den sogleich wieder auffliegenden Männchen aufs Neue (Abb. 6).

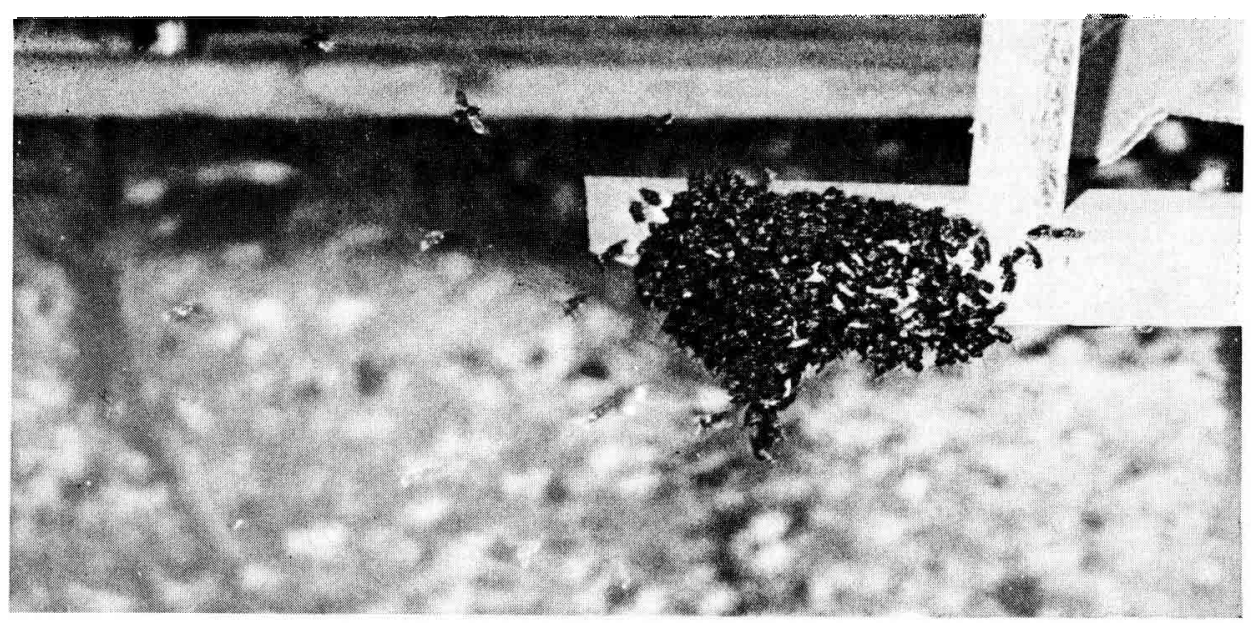

Aвв. 6. - Drohnen-Ansammlung aus knapp 1000 Männchen bei regem Flugbetrieb mit Barbildung, unmitielbar bevor ein Klumpen von Drohnen abreisst und zu Boden fält

FIG. 6. - Drone aggregation of a litte less than 7000 males actively approaching the site and thus forning beards, just before a lump of males teared off and dropped down

Aus Drohnen-Ansammlungen abgefangene Männchen unbekannten Alters fliegen nach der Markierung aus 10-50 m Entfernung sogleich zur Aggregation zurück. Über viele Tage hinweg können sie anschliessend in « ihrer » Ansammlung beobachtet werden, nur wenige finden sich in anderen Männchen-Gruppen vor Nestern in der Nähe ein. Zwei unterschiedlich markierte Drohnen-Aggregationen auf beiden Seiten eines $8 \mathrm{~m}$ breiten, zweistöckigen Laborgebäudes blieben über Wochen hinweg fast unvermischt.

Auf Bienenständen in Brasilien stellten wir zweimal fest, dass Eidechsen die Männchen einer Ansammlung konsumierten. Sie hielten sich unter der Abdeckung unserer Versuchsvölker versteckt (vgl. Nogueira-Neto, 1970).

Bei unvorsichtiger Annäherung eines Menschen fliegen die Drohnen einer Aggregation auf. Greift man, was unschwer möglich ist, eine Handvoll Männchen und drückt sie leicht, so strömen sie einen Duft aus, der Schweissgeruch ähnelt. Eine so riechende Hand veranlasst sitzende Drohnen, sofort aufzufliegen, beun- 
ruhigt Wächterbienen am Flugloch jedoch nicht. Von diesen bei Störung abgegebenes Alarm-Pheromon bewirkt dagegen auch die sofortige Auflösung einer Drohnen-Ansammlung. Alarmierte Männchen fliegen mit den Arbeiterinnen im Zickzack vor dem Nest umher, greifen jedoch nicht wie diese an. Das Nestverteidigungs-Verhalten der Arbeiterinnen ist recht wirkungsvoll; sie stürzen sich in grosser Zahl auf den Feind, setzen sich in den Haaren fest, beissen in die Haut und bekleben den Störenfried mit Kittharz. Die Männchen zeigen keine dieser Reaktionen. Klingt der Alarm ab, so lassen sich auch die Drohnen wieder auf ihrem Sitzplatz nieder.

\section{DISKUSSION}

\section{Wie kommen Drohnen-Ansammlungen zustande?}

Drohnen-Ansammlungen entstehen bei Scaptotrigona postica in Nestnähe. Vom Nest, genauer gesagt vom Nesteingang, werden die Männchen offensichtlich angelockt. Da die Aggregationen nur temporär zu beobachten sind, kommt ein allgemeiner Nestgeruch als Lockreiz kaum in Frage; es muss sich um einen spezifischen, nur zeitweise vom Nest ausgehenden Attraktivstoff handeln.

Unsere Beobachtungen an Ableger-Völkchen machen wahrscheinlich, dass die Drohnen von einem Duftstoff unbegatteter Jungköniginnen, also einen. weiblichen Sexualpheromon angelockt werden. Experimentelle Ableger sind einer Nestfiliale vergleichbar, in die bei einer Kolonieteilung eine oder mehrere Jungköniginnen mit einer grösseren Anzahl Arbeiterinnen umziehen und von dort zum Hochzeitsflug starten (KERR et al., 1962). Vor den Ableger-Kästchen bildeten sich stets nur ein paar Tage lang Männchen-Aggregationen. Die jungen Königinnen gingen anschliessend in Eilage, im Alter von ungefähr zwei Wochen (ENGELS und ENGEls, 1977). Die Drohnen-Ansammlungen stellen also wartende Hoch-zeiterscharen dar.

Aber sprechen die oft langandauernden Drohnen-Ansammlungen vor grossen Kolonien nicht gegen diese Interpretation? Zumindest bei den Beobachtungsvölkern konnte kontrolliert werden, dass in der fraglichen Zeit keine Umweiselung und somit wohl auch kein Hochzeitsflug stattfand. Andrerseits produzieren starke Völker ständig Königinnen, und ein Nest kann gleichzeitig mehrere Jungköniginnen enthalten. KERR et al. (1962) erwähnten bis zu 8, wir fanden einmal 13 in einem Scaptotrigona-Volk. Sie verstecken sich im Involucrum und in leeren Vorratstöpfen. Wenn Jungköniginnen auch in weiselrichtigen Nestern weibliches Sexualpheromon abgeben, würde dies die Bildung manchmal über Wochen hinweg bestehender Drohnen-Ansammlungen vor Altkolonien erklären. Auf Bienenständen mit mehreren grossen Völkern können im übrigen die Aggregationen nicht immer einem bestimmten Nest sicher zugeordnet werden. 
Vergleicht man die Drohnen-Ansammlungen stachelloser Bienen mit den Drohnen-Sammelplätzen der Honigbienen, so ist trotz vieler Übereinstimmungen auf manche prinzipiellen Unterschiede hinzuweisen : Die Drohnen-Sammelplätze der Apinae zeichnen sich durch bestimmte topographische Eigenschaften aus, werden mit Hilfe visueller Orientierung von Drohnen und Königinnen angeflogen und können daher von Jahr zu Jahr immer wieder am selben Platz und über die gesamte Saison hin nachgewiesen werden (ZMARlicki und MORSE, 1963 ; van Praagh und Ruttner, 1975 ; Tribe, 1982). Die Drohnen-Ansammlungen der Meliponinae entstehen an variierenden Neststandorten, die Männchen werden offensichtlich durch ein von unbegatteten Jungköniginnen abgegebenes Pheromon dorthin gelockt und bilden Aggregationen unterschiedlicher Dauer. Dieses ¡Pheromon muss noch identifiziert und als Drohnen-Lockstoff getestet werden.

\section{Wodurch werden Drohnen-Ansammlungen stabilisiert?}

Bei Scaptotrigona postica gibt es offensichtlich keine von Männchen-Aggregationen bevorzugten Lokalitäten. Wir fanden einen Ansammlung nur $25 \mathrm{~cm}$ über dem Erdboden auf niedrigen belaubten Ästen vor einem tiefstehenden Nest und andere über $10 \mathrm{~m}$ hoch in Bäumen. Als Sitzplatz wählten die Männchen häufig glatte Flächen, die jedoch waagerecht oder senkrecht sein können.

Die Reaktionen der Männchen auf Manipulation ihres Sitzplatzes lehren zweierlei. Die Drohnen erlernen die genaue Position ihres Treffpunktes und benutzen beim Anflug wohl Landmarken. Ausserdem markieren sie den Sitzplatz mit einem Pheromon, das wahrscheinlich auch aggregationsfördernd wirkt. Es ist offenbar relativ beständig. Die Verfrachtungsversuche zeigen, dass die Drohnen einer bestimmten Ansammlung diese gezielt wieder aufsuchen. KERR et al. (1962) bestimmten als Rückkehrentfernung $600 \mathrm{~m}$. Wie Experimente mit gezeichneten Männchen weiterhin belegen, ist die Zusammensetzung einer Aggregation auch dann recht konstant, wenn in der Nähe andere Ansammlungen bestehen.

Von entgegengesetzter Wirkung wie das Drohnen-Aggregations-Pheromon ist der Männchen-spezifische Alarmduft. Er veranlasst ein sofortiges Auffliegen der Drohnen einer Ansammlung, alarmiert jedoch Arbeiterinnen nicht. Deren eigenes Alarmpheromon (vgl. BLuM, 1981) warnt hingegen auch die Männchen. Drohnen-Pheromone wurden bei stachellosen Bienen bislang noch nicht identifiziert (vgl. KeEPING et al., 1982 ; FrANCKe et al., 1983).

\section{Welche Rolle spielen Drohnen-Ansammlungen}

im Paarungssystem stachelloser Bienen?

Die Strategie, ein paarungsbereites (virginelles) Weibchen vor dem Nest zu erwarten, ist bei den Männchen vieler Arten solitärer Bienen (BARrows, 1978) 
und Hummeln (ALCock et al., 1978) weit verbreitet. In diesen Fällen wird zumeist der Warteraum von einzelnen Männchen als Mikro-Territorium verteidigt (Barrows, 1976). In den Drohnen-Ansammlungen stachelloser Bienen sind dagegen keinerlei Revier- oder Rivalen-Streitigkeiten zu beobachten. Worin das Konkurrenzverhalten der «friedlich» am gleichen Ort harrenden Männchen besteht, kann sicher erst diskutiert werden, wenn Beobachtungen über natürliche Kopulationen vorliegen. Die wenigen vorliegenden Untersuchungen über Begattungszeichen und Spermienzahlen (KERR et al., 1962) sowie über Kopulation unter kontrollierten Bedingungen (CAMARGO, 197 ) weisen im übrigen auf Einfachpaarung hin.

Nach Verlassen des Nestes vagabundieren die Drohnen von Scaptotrigona postica offensichtlich, was dazu führt, dass sie später nicht in Ansammlungen vor ihrem Muttervolk zu finden sind. Dies belegen einmal die Farbmarkierungen frischgeschlüpfter Männchen in Beobachtungsvölkern in Brasilien, zum anderen erklärt es das Ausbleiben grösserer Drohnen-Ansammlungen vor unseren Versuchsvölkern in Deutschland. Ein Zuwandern von anderen Neststandorten war hier nicht möglich, die von unseren bis zu 3 Kolonien zahlreich produzierten Drohnen wanderten ständig ab. Es muss in Erwägung gezogen werden, ob das Verbleiben von Männchen bei der Mutterkolonie auch durch «kin recognition » verhindert wird.

Drohnen-Ansammlungen vor einem Volk stachelloser Bienen bestehen also überwiegend aus Fremddrohnen. KerR et al. (1962) fanden auch, dass die Aggregationen bei 4 Kolonien sich nicht aus den eigenen Männchen bildeten.

Beim Schwärmen der Honigbienen kann das neue Volk sich in erheblicher Enfernung vom alten ansiedeln. Dies ist bei stachellosen Bienen nicht möglich ; da ein Filialnest über längere Zeit durch Transport von Baumaterial und Vorräten aus der Mutterkolonie aufgebaut wird, kann es nur in deren Nachbarschaft gegründet werden (Nogueira-NeTo, 1954 ; Jui.IANI, 1972 ; Wille und Orozco, 1974). Die Populationsmischung erfolgt somit bei den Meliponinae ausschliesslich durch Migration der Drohnen. Wenn eine Jungkönigin dann mit einem Männchen aus der grossen Schar wartender Freier einer Aggregation kopuliert, ist es mit grosser Wahrscheinlichkeit kein Bruderdrohn.

Die Drohnen-Ansammlungen stachelloser Bienen sind somit Bestandteil eines Paarungssystems, das Geschwisterpaarung weitgehend verhindert. Sie sind hinsichtlich dieses fortpflanzungsbiologischen Effekts den Drohnen-Sammelplätzen der Honigbienen vergleichbar, kommen jedoch auf völlig andere Art und Weise zustande. Da die Paarungs-Strategie der Männchen, vor dem Nest auf ein Weibchen zu warten, bereits bei solitären Apiden entwickelt wurde, kann gefolgert werden, dass in der Evolution der hoch-eusozialen Bienen das Paarungssystem der Meliponinae weniger Abwandlungen erfuhr als das der Apinae. 


\section{DANKSAGUNG}

Wir danken unseren brasilianischen Kollegen Lionel S. GoNÇALves, Warwick E. KERR und Ronaldo Zucchi, die uns wiederholt Gastfreundschaft in ihren Labors gewährten und uns in Ribeirāo Preto ebenso wie in Deutschland stets mit Versuchsvölkern versorgten. Ein Teil der Sachkosten wurde aus DFG-Mitteln finanziert.

\section{RESUME \\ RASSEMBLEMENTS DE MAIES PRES DES NIDS DE L'ABEILLE SANS DARD, SCAPTOTRIGONA POSTICA}

On passe en revue rapidement la biologie de la reproduction des abeilles sans dard. Elles ont fait l'objet de peu de travaux, qui ont porté principalement sur les espèces du Nouvcau Monde. On décrit le rôle des rassemblements de mâles chez Scaptotrigona postica (Trigoninae) d'après des études faites au Brésil sur des colonies sauvages et des colonies de laboratoirc. Des données complémentaires ont été obtenues sur quelques colonies de laboratoire en R.F.A.

Les mâles sont produits tout au long de l'année. Ils quittent la colonje environ 2 semaines après l'éclosion et n'y retournent pas. Ils se nourrissent alors sur les fleurs et se dispersent. Au Brésil les rassemblements de mâles, qui comportent jusqu'à quelques milliers de mâles les uns à côté des autres, se rencontrent au voisinage des nids (Fig. 1, 3, 4 et 6). Certains rassemblements se maintiemnent plusieurs semaines au même endroit. Le marquage de mâles montre qu'ils sont susceptibles de migrer loin de leur colonie natale et qu'ils participent rarement aux rassemblements qui se font devant leur propre colonie. En Allemagne les rassemblements ne comportent souvent qu'un petit nombre de mâles (Fig. 3).

Des nuclei expérimentaux avec reine vierge ont attiré des mâles pendant plusieurs jours (Fig. 5). Les mâles se dispersent avant que la reine ne commence à pondre, ce qui laisse à penser que les rassemblements de mâles sont induits par une phéromone sexuelle femelle. Les rassemblements de mâles servent probablement de lieux de rencontre où les mâles attendent des femelles réceptives.

Les rassemblements de mâles ne se forment que dans la journée, lorsque les conditions atmosphériques sont favorables. L'activité de vol marque un maximum en début d'après-midi. Des mâles qui arrivent essaient parfois de se poser sur des mâles déjà présents, formant ainsi des barbes. Les mâles marqués individuellement ont rejoint régulièrement le même rassemblement. Il n'y a pas de mélange entre les divers groupes de mâles d'une même localité. Des mâles capturés puis relachés à quelque distance sont revenus à leur lieu de rassemblement d'origine. Diverses expériences montrent que les lieux de rassemblement sont marquées d'une odeur. Les mâles réagissent à des phéromones d'alarme secrétées par les ouvrières et semblent posséder aussi un système d'alarme qui leur est propre.

Le comportement des mâles de Scaptotrigona postica est semblable à celui de nombreuses espèces d'abeilles solitaires et de certains bourdons, espèces chez lesquelles les mâles attendent à proximité des sites de nidification de la même espèce, qui servent de lieux de rendez-vous principaux. Du point de vue de l'évolution le système d'accouplement des Meliponinae diffère de celui des abeilles domestiques en semblant moins dérivé que celui des Apinac. Les rassemblements de mâles d'abeilles sans dard et d'abeilles domestiques, bien que se formant de façon totalement différente, doivent être considérés comme des moyens importants d'une stratégie d'outbreeding, destinée à réduire la consanguinité. Comme les nids fils, chez les abeilles sans dard qui essaiment, ne se trouvent qu’à proximité de la colonie mère, la panmixie est réalisée principalement par la migration des mâles. 
On peut conclure qu'au cours de l'évolution de l'organisation eusociale, de nombreux détails de la biologie de la reproduction ont fait l'objet d'une spécialisation chez les Apinae et les Meliponinae.

\section{SUMMARY \\ DRONE AGGREGATIONS NEAR THE NEST OF THE STINGLESS BEE, SCAPTOTRIGONA POSTICA}

A brief review is given of the reproductive biology of stingless bees which has been little studied and mainly in new world species. The role of drone aggregations in the Trigonine, Scaptotrigona postica, is described here based on studies on wild and observation colonies in Brazil. Additional data were obtained from a few lab colonies in Germany.

Males are produced throughout the year. They leave the colony about two weeks after emergence and do not return. Males then feed on flowers and disperse. In Brazil, drone aggregations consisting of a few up to several thousand males positioned close together are found in proximity to nests (Fig. 1, 3, 4 and 6). Some aggregations persist for several weeks at the same site. Marked males. tend to migrate from the area of their natal colony, they seldom participate in aggregations in front of their colony. In Germany aggregations consisted only of very few males (Fig. 3).

Experimental nuclei with virgin queens attracted drones for several days (Fig. 5). The males abandoned before the queen began to oviposit, suggesting that drone aggregations are induced by a female sex pheromone. Drone aggregations probably serve as encounter sites at which males wait for receptive females.

Drone aggregations are formed only during the day when weather conditions are favourable. Flight activity peaks early in the afternoon. Approaching males try to land on already sitting males, thus sometimes forming beards (Fig, 6). Individually marked drones consistently joined the same aggregation. Different male groups in the same locality did not mix. Drones that were captured and released some distance away returned to their original aggregation site. Various experiments indicate that aggregation sites are scent-marked. Drones respond to alarm pheromones released by workers and also apparently have a male-specific alarm system.

Scaptotrigona postica drone behavior is similar to many solitary and some bumblebee species in which males wait in the vicinity of foreign conspecific nest sites, serving as the principal rendezvous places. The mating system in the Meliponinae differs from that of honeybees and is possibly less derived than in the Apinae. Stingless bee drone aggregations and honeybee drone congregations, although formed in a completely different manner, both have to be interpreted as important parts of an outbreeding strategy. As filial nests in stingless bees swarming can only be founded in the proximity of the mother colony, panmixis is effected mainly by drone migration.

It can be concluded that during evolution of eusocial organisation, many details of reproductive biology have been subject to specialization in Apinae and Meliponinae.

\section{LITERATUR}

Adams J., Rothman E.D., Kerr W.E. and Paulino Z.L., 1977. - Estimation of the number of sex alleles and queen matings from diploid male frequencies in a population of Apis mellifera. Genetics, 86, 583-596.

Alcock J., Barrows E.N., Gordh D., Hubbard L.J., Kirkendall L,. Pyle D.W., Ponder T.L. und ZALOM F.G., 1978. - The ecology and evolution of male reproductive behavior in the bees and wasps. J. Linn. Soc. London. Zool., 64, 293-326. 
Barrows E.M., 1976. - Mating behavior in halictine bees : II. Microterritorial and patrolling behavior in $\delta^{\prime} \sigma^{7}$ of Lasioglossum rohweri. Z. Tierpsychol., 40, 377-389.

Barrows E.M., 1978. - Male behavior in Andrena erigeniae with comparative notes. J. Kansas entomol. Soc., 51, 798-806.

BEgo L.R., 1982. - On social regulation in Namotrigona (Scaptotrigona) postica Latreille, with special reference to male production cycles $\left(H_{y m}\right.$., Apidae, Meliponinae). Bolm. Zool. Univ. S. Paulo, 7, 181-196.

BEIG D., 1972. - The production of males in queenright colonies of Trigona (Scaptotrigona) postica. J. apic. Res., 11, 33-39.

Blum M.S., 1981. - Chemical defenses of arthropods. Academic Press, New York.

Camargo C.A. de, 1981. - Mating of the social bee Melipona quadrifasciata under controlled conditions (Hymenoptera, Apidae). J. Kansas entomol. Soc., 45, 520-523.

Eickwort G.C. and Ginsberg H.S., 1980. - Foraging and mating behavior in Apoidea. Annu. Rev. Entomol., 25, 421-446.

Engels W. und Engel.s E., 1977, - Vitellogenin und Fertilität bei stachellosen Bicnen. Insectes soc., 24, 71-94.

Francke W., Schröder W., Engers E. und Engels W., 1983. - Variaton in cephalic volatile substances in relation to worker age and behavior in the stingless bec, Scaptotrigona postica. Z. Naturforsch., 38c, 1066-1068.

Imperatriz-Fonseca V.L., 1977. - Studies on Paratrigona submida (Moure) (Hymenoptera, Apidae, Meliponinae) - II. Behavior of the virgin queen. Bolm. Zool. Univ. S. Paulo, 2, 169-182.

IMPeratriz-Fonseca V.L., 1978. - Studies on Paratrigona subnuda (Moure) (Hymenoptera, Apidae, Meliponiae) - III. Queen supersedure. Bolm. Zool. Univ. S. Paulo, 3, 153-162.

Imperatriz-Fonseca V.L., Oliveira M.A.C. de e Iwama S., 1975. — Notas sobre o compartamento de rainhas virgens de Plebeia (Plebeia) remota Holmberg (Apidae, Meliponinae). Ciênc. e Cult., 27, $665-669$.

Juliani L., 1962. - O aprisionamento de rainhas em colônias de Trigonini. Bol. Univ. Paraná, 20, $1-11$.

Juliani L., 1972. - A descricão do ninho e alguns dados biológicos sobre a abelha Plebeia julianii Moure, 1962 (Hymenoptera, Apidae). Rev. Bras. Entomol., 12, 31-58.

Keeping M.G., Crewe R.M. and Field B.I., 1982. - Mandibular gland secretions of the old world stingless bee, Trigona gribodoi Magretti : Isolation, identification and compositional changes with age. $J$. apic. Res, $21,65-73$.

Kerr W.E. e Krause W., 1950. - Contribuição para o conhecimento da bionomia dos Meliponini. Dusenia, 1, 275-282.

KerR W.E., Zucchi R., Nakadaira J.T. and Butolo J.E., 1962. - Reproduction in the social bees (Hymenoptera : Apidae). J.N. York entomol. Soc, 70, 265-276.

Michener C.D., 1974. - The social behavior of the bees. Harvard Univ. Press, Cambridge.

MourE J.S., 1961. - A preliminary supraspecific classification of the old world Meliponinae bees. (Hym. Apoidea). Stud. Entomol. Rio de Janeiro, 4, 181-242.

Nogueira-Neto P., 1954. - Notas bionômicas sobre Meliponíneos : Ill. Sobre a enxameagem. Arq. Mus. Nac. Rio de Janeiro, 42, 419-451.

Nogueira-Neto P., 1958, - Rainhas prisoneiras. Chácaras e Quintais, São Paulo, $97,477$.

Nogueira-Neto P., 1970. - A criação de abelhas indígenas sem ferrão. Chácaras e Quintais, São Paulo.

Praagh J.P. van and Ruttener F., 1975. - Drone orientation; optical environment at drone congregation area. Proc. 25. Int. Apimondia-Congr. Grenoble, 275-276.

RUTINER F., 1956. - The mating of the honeybee. Bee World, 37, 2-15 und 23-24. 
RUtTner F. und RUT'rner H., 1972. - Untersuchungen über die Flugaktivität und das Paarungsverhalten der Drohnen. 5. Drohnensammelplätze und Paarungsdistanz. Apidologie, 3, 203-232.

SAKagami S.F., 1966. - Techniques for the observation of behavior and social organization of stingless bees by using a special hive. Papéis avulsos Dept. Zool. São Paulo, 19, 151-162.

SAKagami S.F., 1982. - Stingless bees. In : HermanN, H.R. : Social insects vol. 3, $362-423$. Academic Press, New York.

Schwarz H.F., 1948. - The stingless bees (Meliponince) of the western hemisphere. Bull. Am. Mus. Nat. Hist., 90, 1-546.

Silva D.L.N., Zucchi R. and Kerr W.E., 1972. - Biological and behavioral aspects of the reproduction in some species of Melipona (Hymenoptera, Apidae Meliponinae). Anim. Behav., 20, $123-132$.

Terada Y. 1972. - Enxameagem em Frieseomelitia raria Lep. (Hymenoptera, Apidae). In : Homenagem a W.E. KerR, Rio Claro.

Terada Y., 1974. - Contribuição ao estudo da regulação social em Letrotrigona muelleri $e$ Frieseomelita varia (Hymenoptera, Apidae). Tese de Mestrado, Univ. de Ribeirāo Preto.

Tribe G.D., 1982. - Drone mating assemblies. S. African Bee J., 54, 99-100, $103-112$.

Wille A., 1983. - Biology of the stingless bees. Annu. Rer. Enomol, 28, 41-64.

Wille A. and Orozco E., 1974. - Observations on the founding of a new colony of Trigona cupira (Hymenoptera, Apidae) in Costa Rica, Rev. Biol. Trop., 22, 253-287.

WILson E.O., 1971. - The insect societies. Harvard Univ. Press, Cambridge.

Woyke J., 1963. - What happens to diploid drone larvae in a honeybee colony. J. apic. Res., 2, 73-75.

ZMarlicki C. and Morse R.A., 1963. - Drone congregation areas. J. apic. Res., 2, 64-66. 